\title{
Targeting of Periprosthetic Muscles for the Ultrasonographic Screening of Hip Abnormalities in Hip Resurfacing Arthroplasty Patients
}

Hyonmin Choe ( $\sim$ hyonmin@hotmail.com )

Yokohama Shiritsu Daigaku https://orcid.org/0000-0003-4432-2303

Naomi Kobayashi

Yokohama City University Madical Center Department of Orthopaedic Surgery

Hiroyuki lke

Yokohama City University Department of Orthopaedic Surgery

Daigo Kobayashi

Yokohama City University Department of Orthopaedic Surgery

Shintaro Watanabe

Yokohama City University Department of Orthopaedic Surgery

Shota Higashihira

Yokohama City University Department of Orthopaedic Surgery

Akio Otoshi

Yokohama City University Department of Orthopedic Surgery

Kazuma Miyatake

Yokohama City University Department of Orthopaedic Surgery

Yutaka Inaba

Yokohama City University Department of Orthopaedic Surgery

\section{Research article}

Keywords: Hip resurfacing arthroplasty, Ultrasonography, MRI, periprosthetic muscles

Posted Date: March 17th, 2020

DOI: https://doi.org/10.21203/rs.3.rs-17538/v1

License: (c) (i) This work is licensed under a Creative Commons Attribution 4.0 International License.

Read Full License 


\section{Abstract}

Background: Hip resurfacing arthroplasty (HRA) patients require subsequent annual screening for possible post-operative complications. Ultrasonography is an established method for this purpose but lacks a screening protocol for hip joints. The purpose of this study was to evaluate the accuracy of ultrasonography for detecting post-operative complications in HRA patients using a screening protocol that specifically targets periprosthetic muscles.

Methods: We enrolled 45 hips from 40 HRA patients with mean age of 50 years at the time of surgery and a mean follow-up period of 8.2 years. MRI and ultrasonography scans were simultaneously conducted at follow-up. The ultrasonography assessments were conducted on the anterior, lateral, and posterior part of the hip that targets iliopsoas, sartorius, rectus femoris, fascia tensor, short rotators, and gluteus minimus, medius, and maximus. The accuracy of diagnosing postoperative abnormalities and the detectability of periprosthetic muscles were compared between these two modalities.

Results: Both MRI and ultrasonography detected an abnormal region in 8 cases comprising 2 infections, 2 pseudotumors, and 4 patients with greater trochanteric bursitis. Among these cases, 4 hips required implant removal. The increase of anterior space, measured as the distance between the iliopsoas and resurfacing head, was a good indicator for the abnormal mass in these 4 HRA cases. In the assessment of periprosthetic muscles, MRI showed a much lower detectability than ultrasonography in the iliopsoas (6.7\% vs $100 \%)$, gluteus minimus ( $6.7 \%$ vs $88.9 \%)$, and short rotators ( $8.8 \%$ vs $71.4 \%$ ) due to implant halation.

Conclusion: With a screening protocol that targets periprosthetic muscles, ultrasonography can detect postoperative complications as effectively as MRI assessments in HRA patients. Moreover, ultrasonography has superior detectability in the periprosthetic muscles of HRA patients, indicating its utility for the screening of small legions in these cases which may not be detectable by MRI.

\section{Background}

Total hip arthroplasty (THA) is an effective surgical treatment for painful hip disorders with good clinical short- and long-term outcomes but there is always a risk of several post-operative complications, for instance, infections, dislocations, and adverse reaction metal debris (ARMD) (1). Metal on metal (MOM) THA methods, including hip resurfacing arthroplasty (HRA), have been favorable surgical options in the past but have had a high complication rate due to ARMD which can have devastating effects (2-6). Although incidence reports with HRA are much lower than MOMTHA, annual examinations and screening for asymptomatic pseudotumors are required in all HRA patients (7-9).

Measurements of metal ions are a recommended screening method for possible ARMD, but can be prohibitively costly if not covered by national health insurance (10). MRI or CT scans are universal imaging modalities for the screening of pseudotumors (7-9,11-14) but implant halation prevents the careful assessment of periprosthetic soft tissues with these methods, which is problematic in the case of 
implant-related complications that mainly originate in these tissues. Recent improvements to the resolution in ultrasonography have enabled a more precise evaluation of various parts of body and the utility of this approach has been demonstrated for the screening of ARMD in MOMTHA patients $(7-9,14)$. The advantages of ultrasonography in these instances include its cost effectiveness, availability, rapidity, non-use of radiology and minimal effects on metal implants. On the other hand, a major drawback of ultrasonography for the screening of ARMD is the lack of an established protocol in hip joints.

In our current study, we utilized a standardized ultrasonography protocol for the assessment of periprosthetic muscles at the annual follow-up screen in HRA patients and compared the findings with those obtained by MRI. Because many HRA-related complications originate in periprosthetic soft tissues, the purpose of our investigation was to evaluate the accuracy of ultrasonography for the detection of such abnormalities, including ARMD.

\section{Merthods}

This retrospective study was approved by our institutional ethics review board (2019-322). Patients who did not wish to participate or were unable to undergo either MRI or ultrasound were excluded from this study. Among the 76 hips in 64 patients who underwent HRA between 2009 and 2015 at our hospital, 45 hips in 40 patients attended regular follow-ups for more than 3 years and were thus eligible for a comparison of imaging findings between MRI and ultrasonography for possible prosthetic abnormalities. All 40 patients underwent an MRI assessment every 3-5 years and an annual ultrasound. An ultrasound was conducted within 3 months of an MRI assessment in each subject. Among the 40 patients ( 45 hips) that were analyzed, 4 patients ( 4 hips) required revision surgery due to ARMD or periprosthetic joint infection (PJI).

The ultrasonography assessments were conducted on the anterior, lateral, and posterior part of the hip. In terms of the protocol, the ultrasound screen commenced with an assessment of the anterior part in the supine position, lateral part in the lateral position, and the posterior part in the prone position. These periprosthetic assessments consisted of a careful observation of the joint capsule, iliopsoas, sartorius and rectus femoris in the anterior part, fascia tensor, gluteus medius, and gluteus minimus in the lateral part, and gluteus maximus and short rotators in the posterior part (Figure 1). Each peri-prosthetic muscle was observed using the same protocol and the following anatomical landmarks: the anterior superior iliac spine (ASIS), anterior inferior iliac spine (AIIS), and femoral artery for the anterior assessment (Figure 1); ASIS and the greater trochanter for the lateral assessment; and the GTR and ischium tuberosity for the posterior assessment (Figure 2). During assessment of anterior part in the supine position, the distance from the resurfacing head implant to the iliopsoas muscle was measured in the long axis view to quantify the presence or absence of the mass region in the anterior part (Figure 1). All assessments were conducted from the height of the ASIS to the lesser trochanteric. SNiBLE (Konica Minolta, Tokyo, Japan) was utilized for these assessments except for the 4 cases requiring revision surgery for whom a LOGIQ 7 device (GE Healthcare, IL) was utilized for the ultrasonography. 
MRI scans were performed using a 12 channel 1.5-T MR unit (MAGNETOM symphony; A Tim system, Siemens, Germany). Axial and coronal T1 sequence images were obtained with the following parameters: a repetition time (TR) of $500 \mathrm{~ms}$, echo time (TE) of 8.3 minutes, echo train length (ETL) of 3, receiver band width (RBW) of $195 \mathrm{~Hz} / \mathrm{px}$, matrix of axial $\triangle 230 \times 256$ and coronal $\triangle 256 \times 320$, field of view (FOV). from 280 to $340 \mathrm{~mm}$ depending on the patient size, 1 excitation, and a slice thickness from 4 to $506 \mathrm{~mm}$ depending on the patient size. Axial and coronal STIR sequence images were also obtained under the following conditions: TR of $6000 \mathrm{~ms}$, TE of 86 minutes, ETL of 15, RBW of $200 \mathrm{~Hz} / \mathrm{px}$, matrix of $224 \times 320$, FOV from 280-340 $\mathrm{mm}$ and a slice thickness of 4-6 $\mathrm{mm}$ within the total acquisition time of 20 minutes. All MRI images were analyzed by the same surgeon $(\mathrm{HC})$ separately from the ultrasonography. Each periprosthetic muscle was assessed by MRI using a cross sectional image of T1 and STIR from the height of the ASIS to the lesser trochanterics (Figure 3). In the patients that received multiple MRI assessments, the latest assessment or the closest one to the occurrence of complications was chosen for our investigation. The definitive diagnosis of a periprosthetic abnormality was made using MRI findings. The results from our assessments of peri-prosthetic abnormalities on MRI scans were then compared to the findings obtained using ultrasound.

During the follow-up period, the Harris Hip Score (HHS) was noted for the assessment of hip function clinical scores. If there were no abnormal findings on the MRI or unusual pain (i.e. below 40 points in the HHS pain section), the patients were regarded as having made normal progress after the HRA. The diagnosis of ARMD were based on the detection of a pseudotumor on the MRI and through a histopathological assessment of intraoperative tissues with the exclusion of infection (15). PJI was diagnosed based on cultures and histopathological assessment (16). Any other abnormal hip pain was carefully assessed by clinical examination including ultrasonography guided Xylocaine injection.

\section{Statistical analysis}

The accuracy of ultrasound for the detection of abnormal findings in the HRA cohort was determined by calculating its sensitivity and specificity by comparison with MRI findings. The detectability of periprosthetic muscles was compared between ultrasonography and MRI and statistical analyses were conducted using the Fisher's exact test. All tests were reported as significant if the $p$ value was less than 0.05 .

\section{Results}

The mean age of the current study patients was 50 years (range, 34-64 years). The mean Harris hip score was significantly improved from 55 points (mean pain score, 20 points) to 98 points (mean pain score, 43 points) at 1 year post-operation and to 96 points with a mean pain score of 41 points at the final followup (Table 1). During the mean follow-up period of 8.6 years (range, 4.1-10.7), a periprosthetic abnormal mass region of a pseudotumor $(17,18)$ was detected in 4 cases by both MRI and ultrasonography. Among these affected patients, 2 cases were diagnosed with ARMD and 2 with PJI (Table 1). All abnormalities were detectable by ultrasonography as an apparent free echoic spaces in the anterior part of the implant 
(Figures 4 and 5). The accuracy of ultrasonography for the detection of a pseudotumor thus had a sensitivity and specificity of $100 \%$. The distance from the implant to the iliopsoas measured by ultrasonography in the ARMD or PJI cases was significantly longer than in the other cases (mean lengths of $25.8 \mathrm{~mm}$ and $4.0 \mathrm{~mm}$, respectively, $\mathrm{p}<0.01$; Figure 4 ).

Among the 41 non-pseudotumor hips in our present HRA series, 4 cases were diagnosed with greater trochanteric bursitis through the detection of fluid accumulation on an MRI scan with sharp local pain recorded on the greater trochanter. In these 4 patients, a low echoic region around the greater trochanteric was observed on ultrasonography (Figure 6). In one case, iliopsoas impingement was diagnosed through clinical symptoms that included hip flexion pain which immediately improved after an ultrasonography guided xylocaine injection. No structural abnormality was observed on the iliopsoas by either MRI or ultrasonography in this case. Overall, both MRI and ultrasonography detected a periprosthetic abnormality in 8 out of 9 hips that manifested unusual pain after the HRA procedure.

In our assessments of periprosthetic muscles, MRI could produce a clear image of the iliopsoas in $6.7 \%$, sartorius in $97.8 \%$, rectus femoris in $44.4 \%$, gluteus minimus in $6.7 \%$, gluteus medius in $89 \%$, gluteus maximus in $100 \%$ and short rotators in $8.8 \%$ of the patients (Figure 6 ). These data indicated that the iliopsoas, gluteus minimus, and short rotators are not clearly detectable by either T1 or STIR MRI images in most HRA cases, as shown in Figure 3. Notably however, ultrasonography enabled us to assess a complete and clear image of the iliopsoas, sartorius, rectus femoris, gluteus medius, and gluteus maximus with $100 \%$ detectability. However, neither the gluteus minimus nor short rotators could be clearly visualized in some cases and the detectability was reduced to $88.9 \%$ and $72 \%$, respectively (Figure 6). Hence, in comparison with MRI findings, ultrasonography showed greater utility in the assessment of the anterior and posterior parts of the periprosthetic muscles in HRA patients and thus the possibility of making a more precise assessment of periprosthetic abnormalities than MRI (Figure 6).

\section{Discussion}

Ultrasonography is an increasingly prevalent diagnostic modality in the orthopedic field due to advances made in the image resolution. In addition to its rapidity and convenience, an advantage of ultrasonography for the screening of abnormalities in THA patients has been its capacity to closely evaluate periprosthetic abnormalities that can be masked on an MRI or CT scan by metal halation. The early detection of implant related complications is vital in MOM-THA patients to avoid periprosthetic soft tissue damage due to $\operatorname{ARMD}(13,19,20)$. In addition, an abnormal periprosthetic reactions may be presenting through subtle symptoms (21). As represented by ARMD, most implant related complications originate in periprosthetic soft tissues. Therefore, periprosthetic area need to be carefully assessed in MOM THA cases in image screening. Several previous studies have demonstrated the efficacy of ultrasonography in diagnosing ARMD, but no prior report has proposed a ultrasonographic screening protocol for periprosthetic area in HRA patients $(7-9,14)$. Hence, the major goal of our present study was to assess the accuracy of ultrasonography for this purpose using a standardized protocol that focused on the periprosthetic muscles in HRA patients and a comparison with MRI findings. 
In our present analyses, we unified our screening protocol by focusing on anatomical landmarks because a unified record is vital for time course comparisons during a long term follow-up period. Without these landmarks, operator-dependent assessments lack consistency in imaging and interpretation records. The advantage of our current protocol in this regard was that we used anatomical landmarks and surrounding periprosthetic muscle as guideposts that provided a good and consistent orientation of the hip joint. For example, in the anterior evaluations, ASIS and AIIS are good landmarks for identifying the iliopsoas and sartorius muscle, and the rectus femoris. Because these muscles always exist around the hip joint, their evaluation naturally involves observation of the anterior part of the hip joint. In the lateral evaluations, ASIS and the greater trochanter were landmarks for assessing the middle and gluteal muscles and are useful for observing the lateral side of the hip joint. In patients with obesity, posterior evaluations are often difficult but the greater trochanter serves as an index for finding the greater gluteal muscle and external short rotators. The identification of the superior gemellus muscle which attaches to the ischial spine, or the internal obturator muscle that crosses the sciatic notch located just above ischial tuberosity, is an index for observations of the external short rotators and ischial nerve, although thick fat sometimes disturbs the identification of the external short rotator clear edges. In addition, by observing the ischial tuberosity from the greater trochanter, the posterior hip joint can be observed. In our current study patients, most of the periprosthetic muscles except the short rotators could be assessed in a unified image, which is vital for long term follow-up evaluations. The use of anatomical landmarks can also provide better reproducibility and thus more accurate records, even in the severe loosening cases in which the hip center has shifted superiorly due to the central migration of the acetabular component. Notably however, we did not observe any cases of this in our present HRA cohort. In addition, we hypothesize from our current observations that the measured distance between the implant and viable muscle can provide a simple and brief assessment of a possible pseudotumor in HRA patients. In practice, the quantification of the anterior part is easily done by detecting the iliopsoas, although lateral and posterior assessments are still required for the overall evaluation of HRA complications $(7,11)$.

The advantages of MRI are its ability to provide a macroscopic orientation, status, and property of a lesion that cannot be assessed with ultrasonography alone. In other words, MRI is useful for making a definitive diagnosis and lesion type classification, and for a determination of the surgical area. A major drawback of MRI however is that the metal artifact in HRA patients causes a significant loss of the periprosthetic area. The STIR images were tailored for these assessments in our HRA cases, but cannot ensure a qualified assessment of some parts of the periprosthetic area. In addition, the low sensitivity of MRI in detecting periprosthetic muscles indicates the possibility of an underestimation in detecting small inflammatory regions in the screening of asymptomatic patients (21). For these reasons, we considered ultrasonography as a possibly better modality for identifying small legions. Another advantage of ultrasonography over MRI is its utility for conducting direct injections of xylocaine for the detection of pain localization. The diagnosis of ARMD and PJI is important in the postoperative screening of HRA patients, but bursitis or tendinitis may be as prevalent in causing painful THA in these cases and are difficult to diagnose using MRI images alone (1). Ultrasonography is useful however in diagnosing bursitis or tendinitis as represented by the detection of iliopsoas impingement using an echo-guided 
xylocaine test in THA patients. On the other hand, ultrasonography screening still requires radiological assessments for the detection of bony abnormalities represented by osteolysis around the prosthesis as these cannot be visualized by ultrasound.

A notable limitation of our present study was that we only analyzed a small population of HRA patients. Our screening protocol can be improved by assessing larger populations. Another limitation of our current analysis was inability of ultrasound to assess the deep lateral and posterior muscle of the gluteus minimus and the short rotator in some obese patients due to the thickness of the fat layer, or in some cases due to changes in the soft tissue properties caused by the surgical intervention. Although these deep layer muscles were not able to be identified by MRI either, we believe that the use of a probe with a higher resolution and at a lower frequency will help resolve this limitation in the near future.

\section{Conclusion}

We have developed a unified ultrasonographic screening protocol that targets the periprosthetic tissues in HRA patients and provides a screening modality for the detection of ARMD, PJI, and bursitis that is as reliable as MRI and can more precisely detect periprosthetic muscles compared to MRI. Ultrasonography is a therefore a feasible imaging modality for the routine screening of abnormal legions in HRA patients and our unified protocol can provide consistent assessments that may be vital in THA patients who require long-term follow-up evaluations after their operation.

\section{Abbreviations}

THA, total hip arthroplasty; ARMD, adverse reaction to metal debris; MRI, magnetic resonance image; CT, computed tomography; HRA, hip resurfacing arthroplasty; ASIS, anterior superior iliac supine; AllS, anterior inferior iliac supine; F.N., femoral nerve; F.A., femoral artery.

\section{Declarations}

This retrospective study was approved by our institutional ethics review board (2019-322). Patients consent was obtained in the form of opt-out on the website.

H.C, designed the experiments, conducted the experiments, and wrote the paper. N.K, contributed on the design of the experiments, H.I, D.K, S.W, S.H, A, O, K. M contributed on the conduction of experiments and analysis of data, Y.I contributed on design of the experiments and writing of paper. All authors declare no competing interests or funding in relation to this study. The datasets used and/or analysed during the current study are available from the corresponding author on reasonable request.

Acknowledgement:

Not applicable 


\section{References}

1. Henderson RA, Lachiewicz PF, Henderson $₫ R A$, Surgery $O$, Lachiewicz $\otimes P F$. Groin pain after replacement of the hip AETIOLOGY, EVALUATION AND TREATMENT. J Bone Joint Surg Br. 2012;

2. Achten J, Parsons NR, Edlin RP, Griffin DR, Costa ML. A randomised controlled trial of total hip arthroplasty versus resurfacing arthroplasty in the treatment of young patients with arthritis of the hip joint. BMC Musculoskeletal Disorders. 2010;

3. Issa K, Palich A, Tatevossian T, Kapadia BH, Naziri Q, Mont MA. The outcomes of hip resurfacing compared to standard primary total hip arthroplasty in Men. BMC Musculoskeletal Disorders. 2013;

4. Kwon Y-M, Glyn-Jones S, Simpson DJ, Kamali A, McLardy-Smith P, Gill HS, et al. Analysis of wear of retrieved metal-on-metal hip resurfacing implants revised due to pseudotumours. The Journal of Bone and Joint Surgery British volume. 2010;

5. Matharu GS, Pandit HG, Murray DW, Judge A. Adverse reactions to metal debris occur with all types of hip replacement not just metal-on-metal hips: a retrospective observational study of 3340 revisions for adverse reactions to metal debris from the National Joint Registry for England, Wales, Northe. BMC Musculoskeletal Disorders. 2016;

6. Pivec R, Johnson AJ, Mears SC, Mont MA. Hip arthroplasty. In: The Lancet. 2012.

7. Nishii T, Sakai T, Takao M, Yoshikawa H, Sugano N. Is ultrasound screening reliable for adverse local tissue reaction after hip arthroplasty? Journal of Arthroplasty. 2014;

8. Kwon YM, Dimitriou D, Liow MHL, Tsai TY, Li G. Is Ultrasound As Useful As Metal Artifact Reduction Sequence Magnetic Resonance Imaging in Longitudinal Surveillance of Metal-on-Metal Hip Arthroplasty Patients? Journal of Arthroplasty. 2016;

9. Robinson DJ, Lee S, Marks P, Schneider ME. Ultrasound Screening for Adverse Local Tissue Reaction after Hip Arthroplasty. Ultrasound in Medicine and Biology. 2017;

10. Lombardi, A V J, Barrack RL, Berend KR, Cuckler JM, Jacobs JJ, Mont MA, et al. The hip society: algorithmic approach to diagnosis and management of metal-on-metal arthroplasty. J Bone Joint Surg Br. 2012;

11. Hauptfleisch J, Pandit H, Grammatopoulos G, Gill HS, Murray DW, Ostlere S. A MRI classification of periprosthetic soft tissue masses (pseudotumours) associated with metal-on-metal resurfacing hip arthroplasty. Skeletal Radiology. 2012;

12. Yanny S, Cahir JG, Barker T, Wimhurst J, Nolan JF, Goodwin RW, et al. MRI of aseptic lymphocytic vasculitis - Associated lesions in metal-on-metal hip replacements. American Journal of Roentgenology. 2012.

13. J.M. P, E.A. W, S. B, P. B, D. F, T. M. Magnetic resonance imaging findings of adverse reactions to metallic debris (ARMD) of Metal-on-Metal total hip replacements. Skeletal Radiology. 2012.

14. Matharu GS, Janardhan S, Brash L, Pynsent PB, Dunlop DJ, James SLJ. The utility of repeat ultrasound imaging in the follow-up of metal-on-metal hip arthroplasty patients. Annals of the Royal College of Surgeons of England. 2016; 
15. Willert HG, Buchhorn GH, Fayyazi A, Flury R, Windler M, Köster G, et al. Metal-on-metal bearings and hypersensitivity in patients with artificial hip joints: A clinical and histomorphological study. Journal of Bone and Joint Surgery - Series A. 2005;

16. Parvizi J, Gehrke T. Definition of periprosthetic joint infection. Journal of Arthroplasty. 2014.

17. Campbell P, Beaulé PE, Ebramzadeh E, Leduff M, Smet K De, Lu Z, et al. The john charnley award: A study of implant failure in metal-on-metal surface arthroplasties. In: Clinical Orthopaedics and Related Research. 2006.

18. Pandit H, Glyn-Jones S, McLardy-Smith P, Gundle R, Whitwell D, Gibbons CLM, et al. Pseudotumours associated with metal-on-metal hip resurfacings. The Journal of Bone and Joint Surgery British volume. 2008;

19. Nawabi DH, Gold S, Lyman S, Fields K, Padgett DE, Potter HG. MRI predicts ALVAL and tissue damage in metal-on-metal hip arthroplasty. In: Clinical Orthopaedics and Related Research. 2014.

20. Fabi D, Levine B, Paprosky W, Della Valle C, Sporer S, Klein G, et al. Metal-on-Metal Total Hip Arthroplasty: Causes and High Incidence of Early Failure. Orthopedics. 2012;

21. Kwon YM, Ostlere SJ, McLardy-Smith P, Athanasou NA, Gill HS, Murray DW. "Asymptomatic" Pseudotumors After Metal-on-Metal Hip Resurfacing Arthroplasty. Prevalence and Metal Ion Study. Journal of Arthroplasty. 2011;

\section{Figures}




\section{Figure 1}
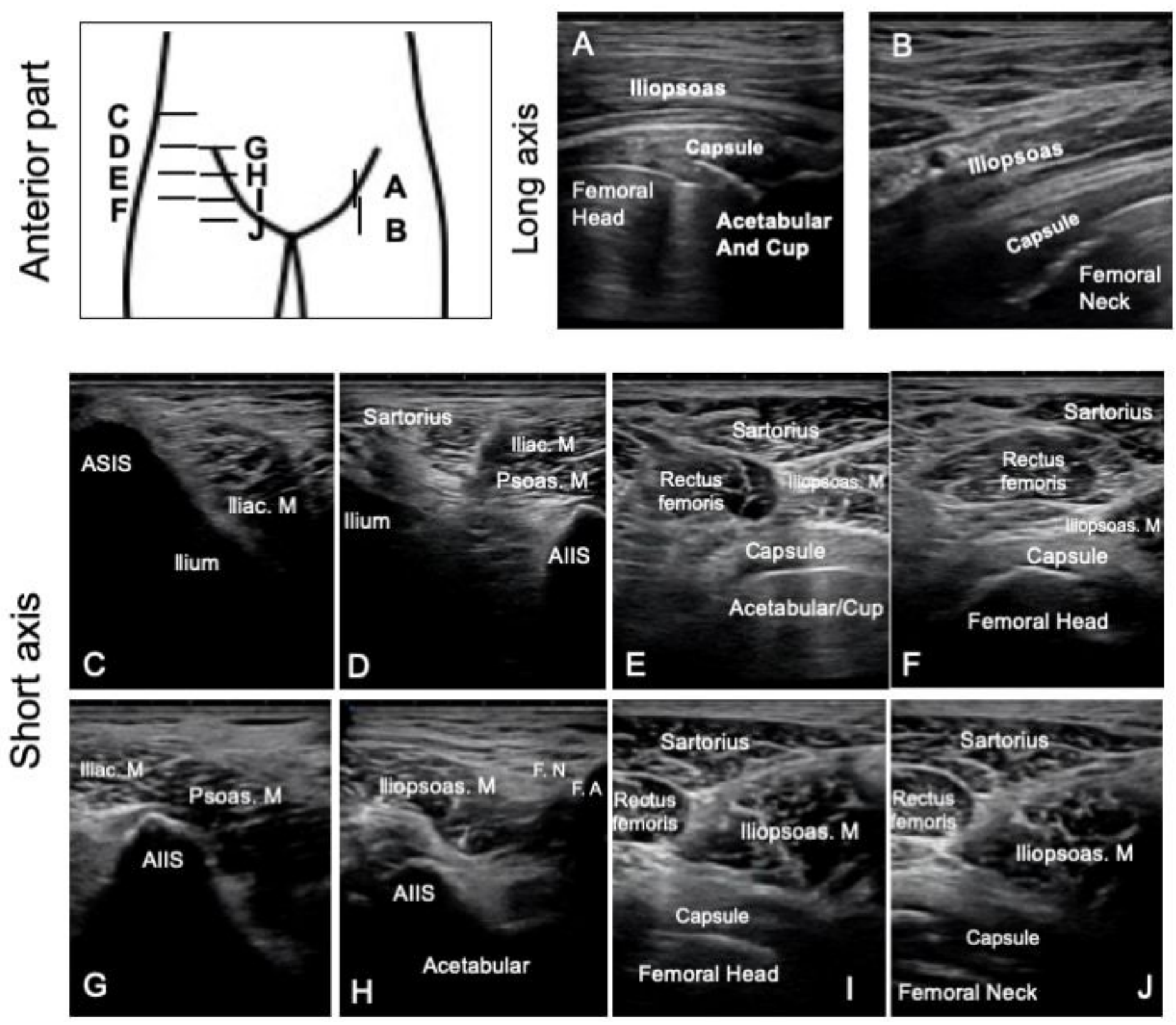

\section{Figure 1}

Representative sonography images of the anterior part in hip resurfacing patients. Periprosthetic hip soft tissues of the anterior part were assessed by longitudinal (A, B) and short axial (C-J) images. In the longitudinal images, the iliopsoas and capsule in front of the acetabular, cup, resurfacing head, and femoral neck were observed (A, B). In the short axial images (C-J), the iliac and psoas muscle, sartorius, rectus femoris, and capsule are observed using the anterior superior iliac supine (ASIS)(C), anterior 
inferior iliac supine (AlIS) (D, G, H), femoral nerve (F.N.) and femoral artery(F.A.)(H) as anatomical landmarks.

\section{Figure 2}
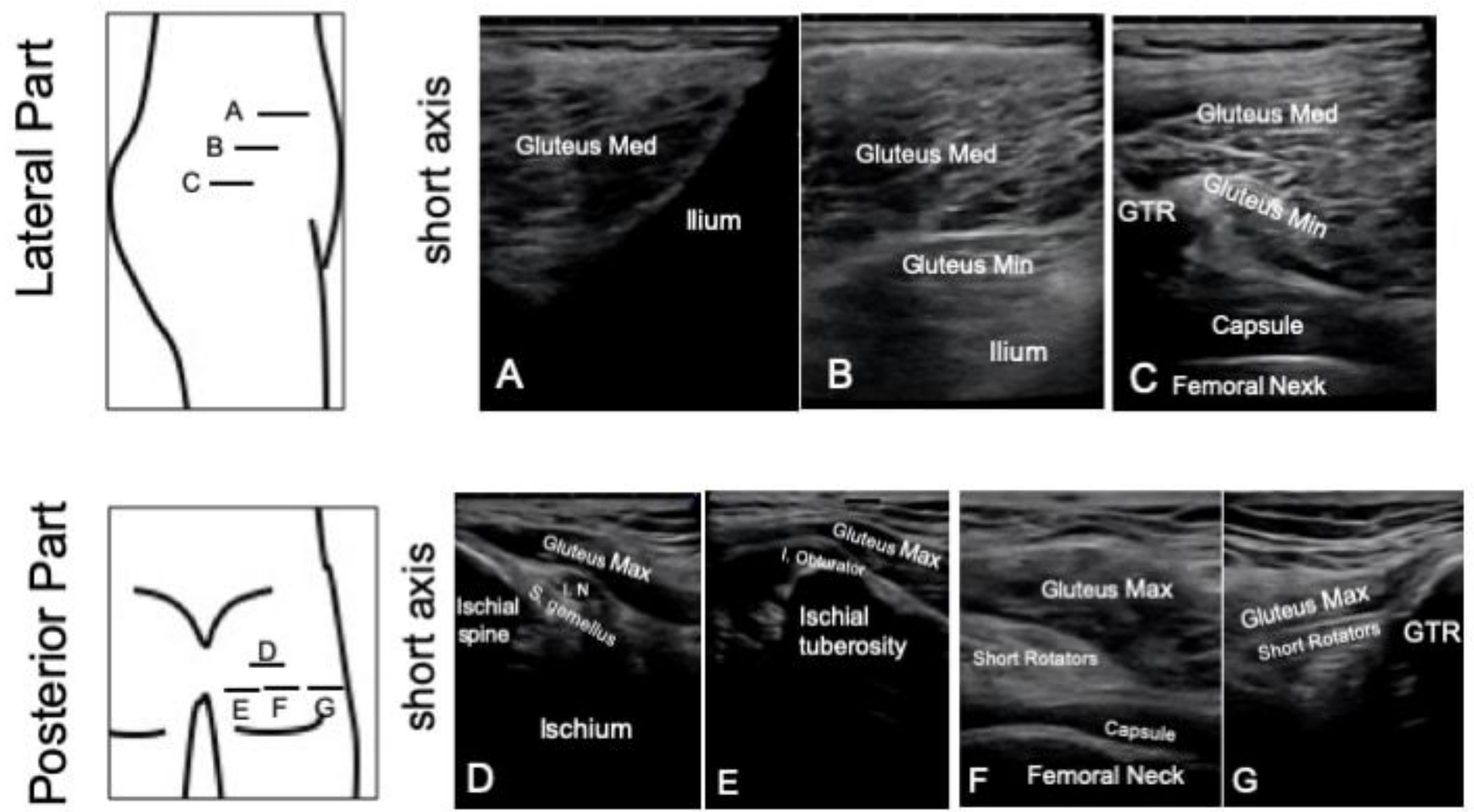

Figure 2

Representative sonography images of the lateral and posterior part in hip resurfacing patients.

Periprosthetic hip soft tissues were assessed using short axial images in the lateral (A-C) and posterior part (D-G). In the lateral assessments, the gluteus medius and minimus were observed using ASIS and GTR as anatomical landmarks (A-C). In the posterior assessment, the gluteus maximus, short rotators and capsule were observed using the ischial tuberosity and GTR as anatomical landmarks (D-G.) Gluteus Med, gluteus medius; Gluteus Min, gluteus minimus; ASIS, anterior superior iliac supine; GTR, greater trochanteric; I.N., ischial nerve; S. gemellus, superior gemellus muscle; I. Obturator, internal obturator muscle. 


\section{Figure 3}
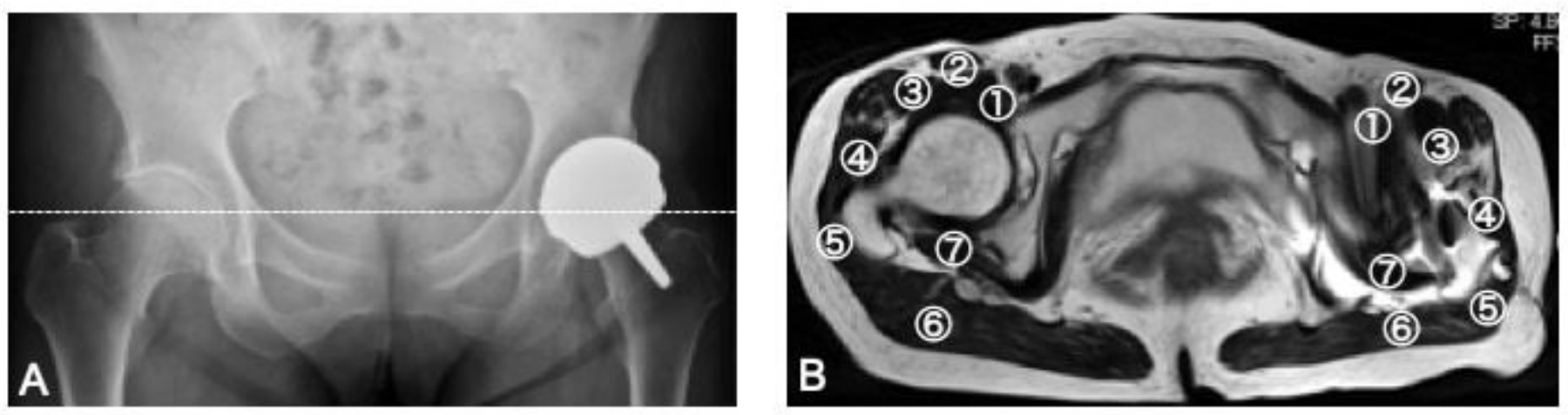

\section{Figure 3}

A representative hip resurfacing case assessed using X-ray and MRI. Apparent complications in this representative patient were not found by hip X-ray (A). The periprosthetic muscles consisted of 1 . iliopsoas, 2. sartorius, 3. rectus femoris, 4. gluteus minimus, 5. gluteus midius, 6 . gluteus maximus, 7 . short rotators which were observed on by axial MRI (B) from the ASIS to lessor trochanteric level on T1 and T2 STIR images. If the contours of the targeting muscles cannot be observed, the muscle is regarded as unassessable. 


\section{Figure 4}

\section{$\mathrm{mm}$}

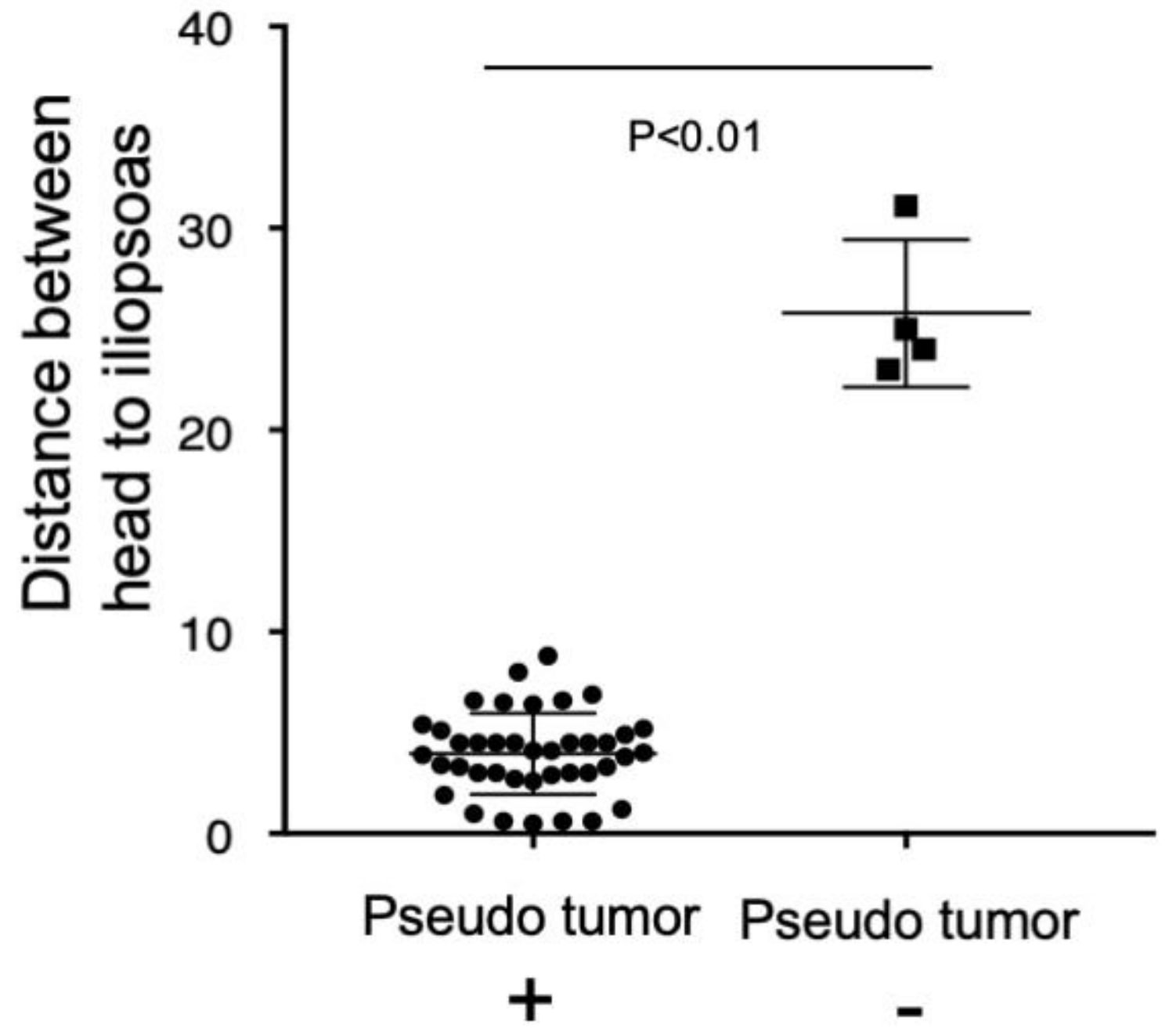

Figure 4

Comparison of the anterior distance between pseudotumor and non-tumor patients. The distances between the resurfacing head and the iliopsoas in the anterior part were measured in all HRA patients by ultrasonography. The mean distance was significantly higher in the pseudotumor patients $(25.8 \mathrm{~mm}$ vs. $4.0 \mathrm{~mm}, \mathrm{p}<0.01)$. 


\section{Figure 5}
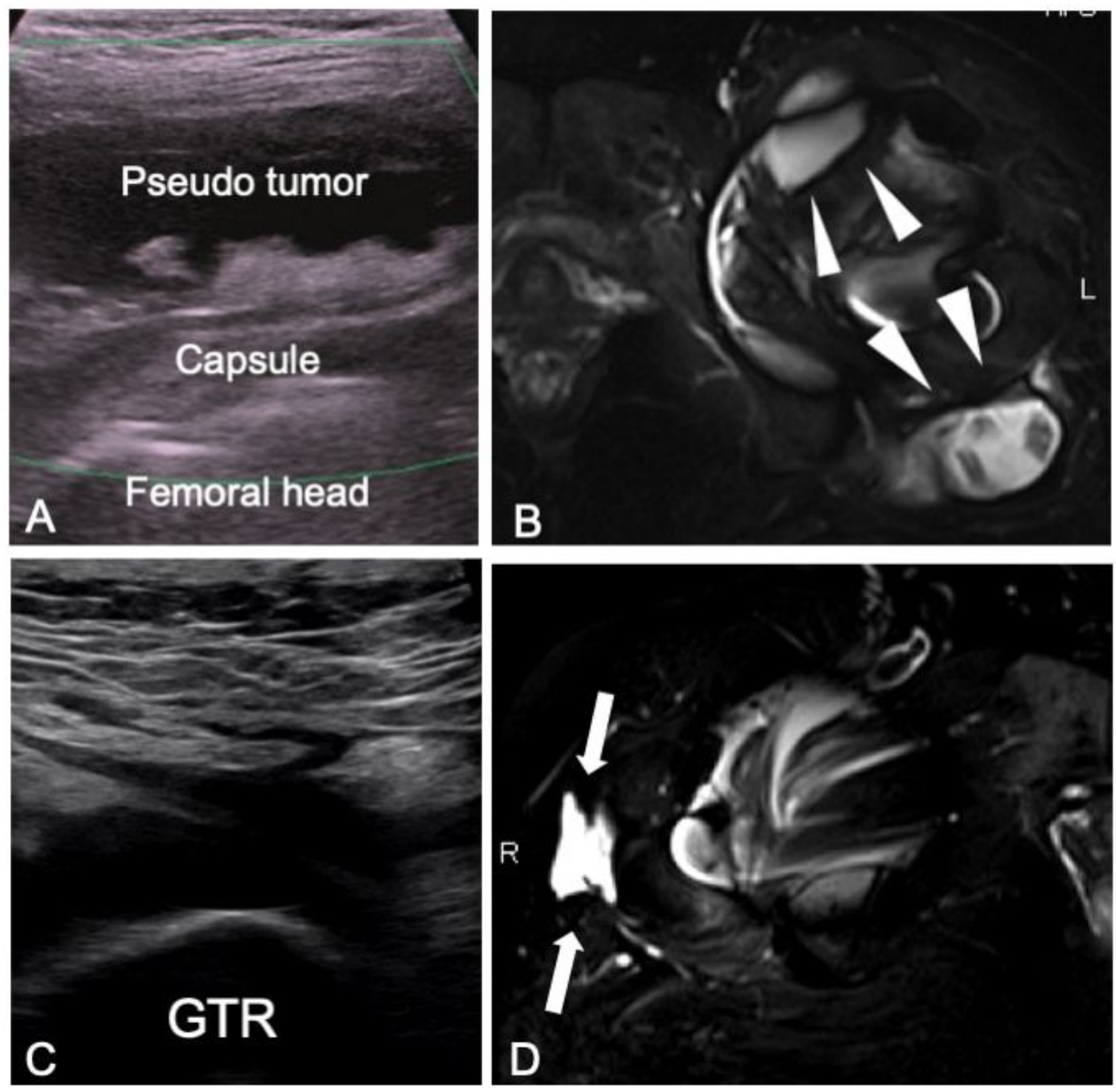

\section{Figure 5}

Representative abnormal images taken using ultrasonography and MRI in the hip resurfacing patients. (A$B)$ : A pseudotumor was detectable in the anterior long axis of the hip by ultrasonography as a free echoic space above the femoral head (A). MRI provided information regarding the area of spread and lesion properties that is vital for the preoperative assessment of the pseudotumor (B). The trochanteric synovium was detected by ultrasonography in a patient with lateral thigh pain as a free echoic space 
located above the greater trochanter (C). MRI also provided information on local fluid collection at the lateral side of the greater trochanter (D).

\section{Figure 6}

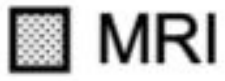

U Ultrasonography

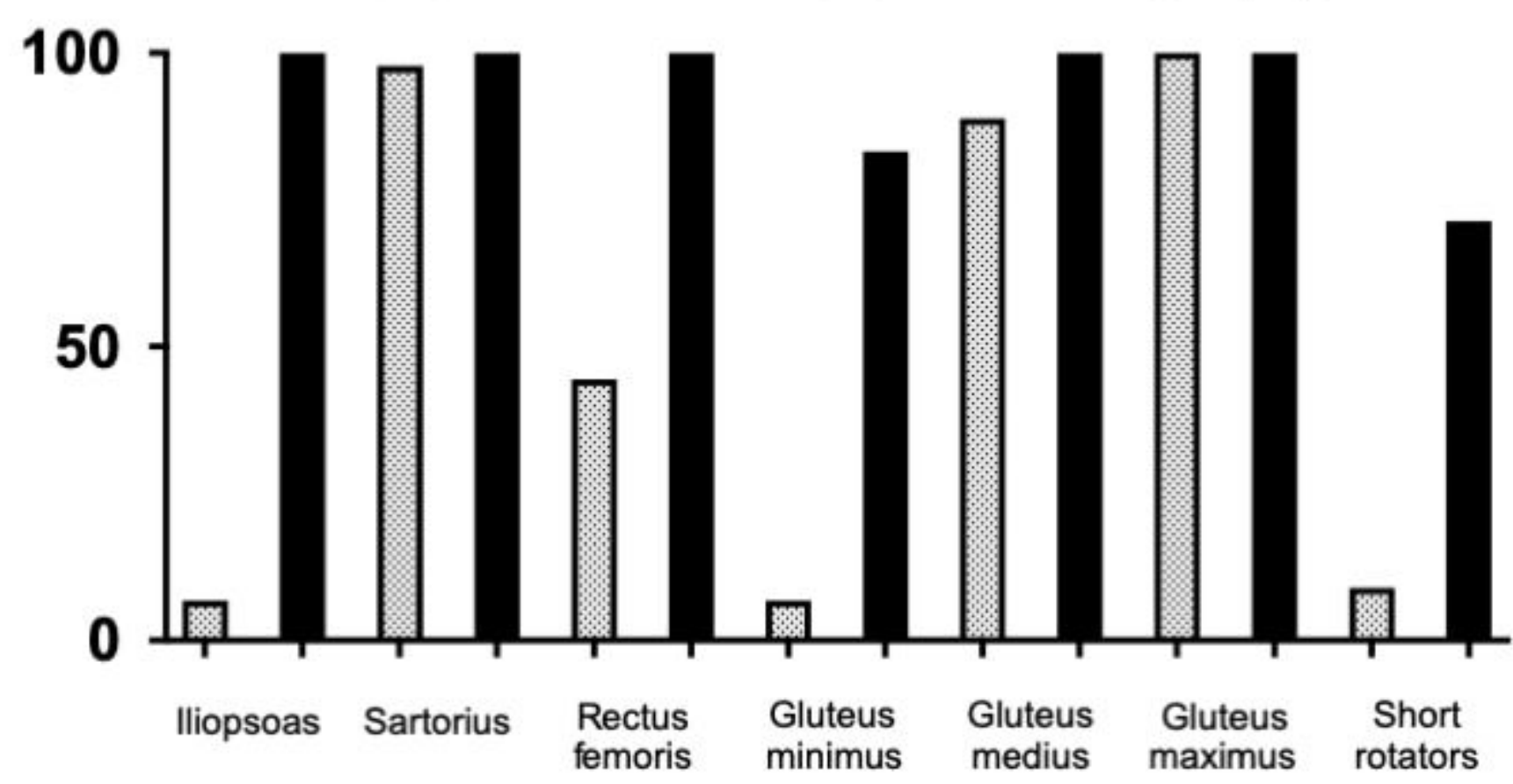

Figure 6

Detectability of periprosthetic muscles in the study cohort by MRI and ultrasonography. In the anterior part, the iliopsoas, sartorius, and rectus femoris were detectable by MRI in $6.7 \%, 97.8 \%$, and $44.4 \%$ of the HRA cases, although ultrasonography showed $100 \%$ of detectability of all of these muscles (A). In the lateral part, the gluteus minimus and medius could be detected in $6.7 \%$ and $89 \%$ of the patients by MRI, and in $88.9 \%$ and $100 \%$ of these cases by ultrasonography. The gluteus maximus and short rotators were detectable in $100 \%$ and $8.8 \%$ of the patients by MRI and $100 \%$ and $71.4 \%$ of the patients by ultrasonography. 